Revista de Psicología Vol. 34 (2), 2016 (ISSN 0254-9247)

\title{
Concepciones de conflictos interpersonales y desarrollo moral en la educación infantil brasileńa
}

\author{
Alia Barrios ${ }^{1}$ \\ Instituto de Educación Superior de Brasilia/IESB; Universidade de Brasilia
}

\begin{abstract}
Este artículo presenta parte de un estudio sobre desarrollo moral realizado en una institución pública de Educación Infantil de Brasilia desde la perspectiva sociocultural constructivista, en el que fueron analizadas las concepciones de nueve educadores sobre el papel de los conflictos interpersonales en el desarrollo moral, a través de su elaboración discursiva y de las estrategias pedagógicas usadas para intervenir en situaciones de conflicto. Los resultados mostraron el predominio de una visión negativa del conflicto por parte de los educadores, así como la necesidad de cambios en esa visión en función de la importancia de los conflictos interpersonales para la construcción de valores morales y de habilidades sociales que permitan la convivencia armónica y democrática.

Palabras clave: conflictos interpersonales, desarrollo moral, educación infantil, perspectiva sociocultural constructivista.
\end{abstract}

\section{Ideas about Interpersonal Conflicts and Moral Development in Brazilian Early Childhood Education}

This paper presents part of a study of moral development conducted in a public early education school in Brasília, Brazil. A sociocultural constructivist perspective was employed to analyze the ideas of nine educators about the role of interpersonal conflict in moral development. Educators provided information about the pedagogical strategies they use to intervene in conflicts. Results show the dominance of a negative view of the conflict and the need for changes in this perspective due to the role that interpersonal conflicts play in building moral values and social skills that enable a harmonious and democratic coexistence. Keywords: Interpersonal conflicts, moral development, early childhood education, sociocultural constructivism.

1 Doctora en Procesos de Desarrollo Humano y Salud por el Instituto de Psicología de la Universidad de Brasilia y docente en el Instituto de Educación Superior de Brasilia y en la Facultad de Educación de la Universidad de Brasilia. Dirección postal: SHCES Quadra 911 Bloco B apto. 304. Cruzeiro Novo, Brasilia-DF, Brasil CEP: 70655-002. Contacto: aliabarrios@gmail.com 


\section{Concepçóes sobre conflitos interpessoais e desenvolvimento moral na Educaçáo Infantil brasileira}

O presente artigo apresenta parte de um estudo sobre desenvolvimento moral realizado numa instituição pública de Educação Infantil de Brasília-DF, Brasil. No estudo, realizado a partir da perspectiva sociocultural construtivista, foram analisadas as concepçóes de nove educadores sobre o papel dos conflitos interpessoais no desenvolvimento moral, através de sua elaboração discursiva e das estratégias pedagógicas usadas para intervir em situaçóes de conflito. Os resultados mostram o predomínio de uma visão negativa do conflito por parte dos educadores, assim como a necessidade de mudanças nessa visão em função da importância dos conflitos interpessoais para a construção de valores morais e de habilidades sociais que permitam uma convivência harmônica e democrática.

Palavras chave: conflitos interpessoais, desenvolvimento moral, educação infantil, perspectiva sociocultural construtivista. 
El estudio fue realizado a partir de la perspectiva sociocultural constructivista, que enfatiza la importancia de los conflictos interpersonales en el proceso de socialización, desarrollo y educación moral del niño, además de ver a la escuela como un contexto central para esos procesos. La escuela es un espacio privilegiado para que los conflictos se conviertan en experiencias constructivas y formadoras de sentimientos como la empatía y el respeto por el otro. Además de eso, la escuela se destaca como un contexto fundamental para el desarrollo humano, de forma general. De acuerdo con Fleer, Heddegard y Tudge (2009), el espacio escolar participa de la transmisión y de la construcción del conocimiento culturalmente organizado, siendo un espacio de socialización privilegiado. Es en el contexto escolar donde el niño tiene posibilidad de establecer interacciones y relaciones complejas con los adultos y con otros niños de diversas edades, niveles de desarrollo y características diferentes. $\mathrm{Y}$ es en el contexto de esas interacciones y relaciones complejas que se construye la moralidad (Branco, 2009; De Vries y Zan, 1998; Ratner, 2002; Rogoff, 2005).

\section{Los conflictos interpersonales en el escenario de la psicología}

El concepto de conflicto ha sido abordado por diferentes áreas de la psicología desde diversas perspectivas y a partir de diferentes modelos teóricos, enfatizando sus dimensiones afectivas, cognitivas y motivacionales. En el contexto de la psicología social, las ideas de Lewin (1935) fueron fundamentales para la comprensión del término. Lewin (1935), explicó el conflicto como una oposición entre fuerzas que tienen valor semejante. Identificó tres tipos de conflictos básicos que se relacionan con el posicionamiento del sujeto frente a situaciones que pueden tener valor positivo o negativo, generando una situación conflictiva en términos internos. De acuerdo con Valsiner y Cairns (1992), dicha perspectiva concentró sus esfuerzos en la comprensión del conflicto 
en términos motivacionales. Perspectivas posteriores de la psicología social han pensado el concepto a partir de las relaciones interpersonal es enfatizando los conflictos entre grupos y en diversos ámbitos institucionales. Temas como causas, intensidad, gestión, formas y habilidades sociales para la negociación y resolución del conflicto hacen parte de las investigaciones y trabajos de esa área (e.g. Bellini-Leite, Vargas \& Ireno, 2012; Martins \& Garcia, 2011; McIntyre, 2007; Oetzel, Dhar \& Kirschbaum, 2007; Sanchez-Burks, Nisbett \& Ybarra, 2000; Ting-Toomey, 1988).

Para la psicología del desarrollo, el estudio de los conflictos que surgen en las interacciones de los niños con sus pares también ha sido fundamental, ayudando a comprender las interacciones sociales 'negativas' de los niños, así como las posibles formas de intervención del adulto ante las mismas (e.g. Buhs, Ladd \& Herald, 2006; Frick, 2011; Kolominskii \& Zhiznevskii, 1992; Ladd \& Troop-Gordon, 2003).

En la psicología del desarrollo, de forma general, el conflicto ha sido conceptuado como una situación de oposición entre las partes integrantes de un sistema interactivo. En la perspectiva tradicional del desarrollo, esa oposición ha sido clasificada en dos formas específicas en función de sus posibles consecuencias para el sistema: el conflicto positivo y el conflicto negativo. El conflicto positivo es aquel en el cual la relación de oposición entre las partes del sistema conduce a la emergencia de nuevos estados en aquel sistema. Ya el conflicto negativo implica un choque de competición excluyente, devastando una parte u otra y, de ese modo, conduciendo a la extinción del todo del cual son partes integrantes. Aunque sea importante considerar las consecuencias de la oposición para el sistema interactivo, dicha clasificación establece una dicotomía excluyente entre los dos tipos de conflictos y no resalta el importante papel que los dos pueden desempeñar en el desarrollo del individuo. Determinadas situaciones de oposición pueden conducir a la extinción del sistema interactivo, pero al mismo tiempo pueden posibilitar mudanzas significativas para las partes integrantes del mismo.

Según Valsiner y Cairns (1992), la visión del concepto de conflicto como 'positivo' y 'negativo' desde una perspectiva dualista y excluyente 
se configura y sustenta a partir de diversos estudios sobre el concepto y a partir de la codificación semiótica del concepto en el lenguaje de sentido común, una vez que los investigadores son, en primer lugar, miembros de una cultura colectiva y, en segundo lugar, científicos.

Además de eso, y también según Valsiner y Cairns (1992), la visión del conflicto en una perspectiva dualista y excluyente se debe a que el estudio del concepto tiene como base el principio de separación exclusiva que procura explicar y entender los fenómenos a partir de sus partes separadas. O sea, partiendo del principio de separación exclusiva, el concepto de conflicto se define por sus partes separadas y en oposición, vistas como diferentes y no interrelacionadas. Su resolución está relacionada a la selección de una de sus partes, lo que lleva, necesariamente, a la eliminación de la otra.

Jares (2006) también se refiere a la visión excluyente o concepción tradicional del conflicto y explica que la misma trae como consecuencia la idea del conflicto como algo que no se debe transformar sino evitar. Para el autor, la mayoría de las teorizaciones sobre el conflicto interpersonal lo definen como sinónimo de incompatibilidad entre personas o grupos, o entre fines y valores que, generalmente, se muestran inconciliables unos con los otros. Esa incompatibilidad muchas veces se toma como sinónimo de formas no-apropiadas de resolución (agresión, violencia, coerción, etc.), que tienden a suprimir el conflicto apuntando para la eliminación del adversario. Concordamos con Jares (2006) cuando especifica que esas formas no-apropiadas de resolución se constituyen medios o estrategias, mientras que el conflicto se constituye un proceso de causas múltiples, que envuelve protagonistas en contextos socioculturales específicos, y que abarca fines y valores que pueden mantener una relación dialéctica.

Aún en el contexto de la psicología del desarrollo, autores como Piaget (1932/1994), Selman (1980) y Wallon (1941/2007) abordan el conflicto desde perspectivas diferenciadas que enfatizan la importancia y el carácter constitutivo del conflicto para el desarrollo humano, de forma general, y para los procesos de socialización y de construcción de la moralidad, de manera más específica. 
Piaget (1964/2006) resalta la dimensión cognitiva, colocando el conflicto como una oposición entre los esquemas o estructuras mentales ya desarrollados y nuevas experiencias, informaciones y percepciones de la realidad. El conflicto cognitivo en la perspectiva piagetiana es fundamental para el desarrollo de nuevos esquemas o estructuras mentales y para el proceso continuo de equilibración y adaptación. En relación al desarrollo de la moralidad, Piaget (1932/1994) resalta el papel de las interacciones sociales y de los conflictos que surgen a partir de ella como oportunidades para el desarrollo de la consciencia moral autónoma. Es en el contexto de las interacciones sociales que el sujeto tiene la posibilidad de descentrarse cognitivamente y ver la realidad a partir de los puntos de vista de otras personas. Esa posibilidad de descentración se construye durante el transcurso de desarrollo del sujeto que empieza a percibir las partes envueltas en la interacción social como dignas de ser respetadas y tratadas igualmente o de forma justa.

Selman (1980) realizó varias investigaciones que son referencias para los estudios y trabajos relacionados con la resolución de conflictos interpersonales entre nińos y/o colegas de la misma edad. El autor elaboró los trabajos de Piaget (1932/1994) sobre el desarrollo de la capacidad de asumir el punto de vista del otro y coordinarlo con el propio punto de vista. De acuerdo con Selman (1980), esa capacidad pasa por niveles que van desde la perspectiva egocéntrica del nińo pequeño hasta el entendimiento interpersonal que puede reflejarse en la conducta y en las relaciones sociales del niño mayor.

Los diferentes niveles de entendimiento interpersonal elaborados y estudiados por Selman (1980) tienen como base dos tipos de interacciones fundamentales para el proceso de socialización del niño. La primera es la negociación, que tiene como objetivo mantener la propia identidad separada de la identidad de los otros. Es decir, el niño consigue considerar el punto de vista de los otros, pero no consigue articularlo a su propio punto de vista, en el sentido de compartir y cooperar con objetivos diferentes de los suyos. La segunda es la cooperación, que tiene como objetivo establecer la posibilidad de reciprocidad con los otros. En ella, el niño consigue considerar el punto de vista de 
los otros y articularlo con su propio punto de vista, modificando el último en función de objetivos comunes que puedan ser compartidos. Para Selman (1980), la negociación no representa el nivel más alto de entendimiento interpersonal, pero es fundamental para el desarrollo de experiencias compartidas y para la cooperación.

Para Wallon (1941/2007), los conflictos tienen un papel fundamental en el proceso de formación de la personalidad y pueden ser comprendidos a partir de la idea de diferenciación o distinción entre el yo y el otro, que es una idea clave en la teoría walloniana (Galvão, 1995). Durante el período del personalismo (de los tres a los seis años), el niño se opone, de manera sistemática, a lo que percibe como diferente de él (el otro o no-yo) en un intento de comprobar la independencia de su personalidad. El conflicto yo-otro también se vive en la adolescenciaenun nivel cualitativamente diferente. Mientras que el niño pequeño vive sus conflictos de manera más emocional, el adolescente intenta argumentar de forma racional sus oposiciones en relación al otro. En la perspectiva walloniana, los conflictos, vistos como situaciones o crisis de oposición, tiene un carácter constituyente y están presentes a lo largo de todo el proceso de desarrollo de la persona. De acuerdo con Wallon (1941/2007), el otro con las fronteras y límites que él coloca, es un compañero constante del yo en la vida psíquica.

Partiendo del papel de los conflictos en la constitución de la personalidad, Wallon (1941/2007) también resalta su importancia en el contexto educativo. Para el autor, varias situaciones de oposición que ocurren en el contexto de la interacción profesor-alumno y alumno-alumno parten de la necesidad de diferenciación del yo del no-yo, siendo tarea del profesor descubrir procedimientos prácticos para lidiar de forma adecuada con la situación. Además de eso, el profesor se constituye algo privilegiado para el ejercicio de la oposición en función de su posición de autoridad, y de la necesidad de autonomía del nińo y del adolescente en proceso de desarrollo. Otras situaciones de conflicto importantes en el contexto educativo son las dinámicas turbulentas que se caracterizan por la dispersión y agitación motora y que generan interacciones desgastantes o entrópicas sin valor constituyente para la personalidad (Galvão, 1995). 
Aunque la psicología y otras áreas de la ciencia hayan cambiado, en el contexto de sus teorías más contemporáneas, la visión del conflicto, el contexto educativo continúa enfatizando la visión dualista, excluyente y negativa del concepto. En el contexto escolar, los conflictos interpersonales son vistos y analizados por la perspectiva de la violencia (Jares, 2006), restringiendo las posibilidades de la escuela como espacio de desarrollo y educación para la convivencia armónica y para la participación social activa. Siendo así, es importante estudiar y comprender las concepciones de los docentes con relación al conflicto y conocer las estrategias de intervención que se originan de ellas. Esos objetivos estuvieron presentes en el estudio que presentamos, pues pensamos que con ese conocimiento será posible contribuir para el desarrollo de modelos pedagógicos que, de hecho, privilegien el desarrollo y educación moral del niño.

\section{Conflictos interpersonales y desarrollo moral en la perspectiva sociocultural constructivista}

En el contexto de la perspectiva sociocultural constructivista, que sirvió de base a nuestra investigación, el conflicto es visto a partir del principio de la separación inclusiva que, conforme presentado por Valsiner (1989, 2012) y Valsiner y Cairns (1992), se refiere a la interdependencia de 'opuestos aparentes' dentro de un mismo sistema interactivo. Los 'opuestos aparentes' se interrelacionan y, al mismo tiempo, mantienen su independencia. De acuerdo con Valsiner y Cairns (1992), el principio de la separación inclusiva permite definir el conflicto a partir de las relaciones articuladas (o interrelaciones) entre las partes de un todo. Relaciones articuladas que posibilitan la visión del concepto en un continuum, así como la visión de las oposiciones entre sus partes y la posibilidad de coexistencia y transformación de una parte en la otra. De manera resumida, podemos decir que a partir del principio de separación inclusiva, conceptos y fenómenos vistos como opuestos se vuelven interdependientes e coexisten (Valsiner \& Cairns, 1992).

Considerando esa visión del conflicto, podemos afirmar que las situaciones de conflicto y/o contradicción son educativas, a medida en que son capaces de producir tensión emocional y colocar al sujeto 
delante de la necesidad de presentar acciones cualitativamente diferentes de las presentadas hasta el momento, impulsando, así, su desarrollo. Sin embargo, es necesario considerar que los conflictos y/o contradicciones que no se explicitan para que sean elaborados pueden convertirse en objeto de tensión emocional entre los sujetos, y transformarse en focos permanentes de malestar, productores de disposiciones negativas que, en general, no conducen al desarrollo (Rey \& Martínez, 1989). O sea, el papel del conflicto en el desarrollo personal puede estar relacionado con la explicitación y la forma de administrar las oposiciones y divergencias.

Partiendo de las ideas anteriores, la perspectiva sociocultural constructivista enfatiza el papel fundamental del conflicto para el desarrollo humano y, especialmente, para el desarrollo moral, entendiendo la moralidad como un fenómeno complejo que envuelve las dimensiones conductual, normativa y motivacional, como explicitadas por Freitag (1997) y por Branco y Barrios (2014). Para las autoras, la moral está relacionada con la acción de los sujetos en el contexto de las interacciones sociales. Esa acción está orientada y puede ser juzgada a partir de un conjunto de reglas y normas que determinan, en nivel sociocultural, lo que se considera adecuado, justo y correcto en la interacción con los otros. Algunas de esas reglas y normas pueden constituirse principios y valores que orientan, de forma más estable, las acciones e interacciones en función de su importancia para la convivencia social. Esas normas y reglas constituyen la dimensión normativa de la moralidad, que presupone un sujeto consciente, capaz de juzgar lo cierto y lo errado, el bien y el mal, lo justo y lo injusto. Por otro lado, la moralidad presupone un conjunto de razones que llevan al sujeto a comportarse de una forma determinada. Las razones, motivos e intencionalidad constituyen la dimensión motivacional, que es esencial para la construcción y comprensión de la moral.

Para la perspectiva sociocultural constructivista, el desarrollo moral es un fenómeno complejo que, en función de su naturaleza social, tiene lugar en el contexto de las relaciones e interacciones que el niño establece con sus colegas y con los adultos responsables por él 
(Barrios, 2013; De Vries \& Zan, 1998, 2003). En función de su naturaleza cultural y, al mismo tiempo, subjetiva, el desarrollo moral ocurre a partir de las experiencias de vida concretas que la persona tiene en el contexto de las prácticas culturales específicas de su cotidiano (Branco, 2009, 2012; Martins \& Branco, 2001; Paolichi, 2007; Ratner, 2002; Rogoff, 2005).

Como colocamos en el inicio del presente texto, la perspectiva sociocultural constructivista enfatiza la importancia de los conflictos interpersonales para el proceso de socialización, desarrollo y educación moral del niño, y ve a la escuela como un contexto central para esos procesos. En el caso específico del contexto escolar, el conflicto está presente con frecuencia, tanto en las interacciones profesor-alumno como en las interacciones alumno-alumno. De acuerdo con DeVries y Zan (1998), el conflicto interpersonal es un elemento presente e importante del ambiente sociomoral de la clase, que debe ser conducido de forma significativa para el desarrollo y la educación moral de los niños. Sin embargo, la idea de la posibilidad de conflictos parece constantemente perturbar al profesor, que pasa a evitarlos como si fueran siempre y exclusivamente negativos (Barrios, 2009; Galvão, 2004; Licciardi, 2010; Lima, 2000).

Autoras como DeVries y Zan (1998) y Corsi (2011) resaltan el significado de diversas estrategias e interferencias educativas a la hora de lidiar con los conflictos interpersonales dentro de la clase. Esas estrategias pasan por la posibilidad de negociación de las divergencias por parte de los propios nińos, el reconocimiento de los sentimientos, deseos y percepciones de los envueltos en la situación de conflicto, la oportunidad de criar y sugerir soluciones adecuadas para la situación de conflicto, y la oportunidad de compensación y restablecimiento del vínculo interpersonal. En todas esas estrategias e interferencias, el educador puede asumir una posición de mediador, en el sentido de ayudar a los niños a partir de su experiencia y, al mismo tiempo, dejar el espacio para que ellos se responsabilicen por sus conflictos y ejerzan la autonomía y creatividad en sus negociaciones. 
Los conflictos interpersonales, cuando negociados y resueltos de forma autónoma y creativa, pueden configurarse experiencias importantes que permiten la descentración por parte de los envueltos. Percibir los sentimientos y necesidades del otro, así como los propios, es un elemento fundamental para la construcción y expresión de sentimientos como la empatía y respeto, que están en la base de la motivación social (Hoffman, 2000). Por otro lado, en las situaciones de conflicto también es posible percibir la necesidad de establecer reglas y normas que orienten la acción en relación al otro y a la convivencia social de forma general.

\section{Método}

La perspectiva sociocultural constructivista presenta una visión de metodología como proceso cíclico de producción de conocimiento, que envuelve diferentes momentos, así como la participación activa del investigador en el proceso de aproximación de los datos. De acuerdo con Branco y Valsiner (1997), la metodología es el proceso de pensamiento de los procedimientos de intervención y de interacción con el fenómeno estudiado. Procedimientos de intervención que pueden ser diversificados, siempre que compatibles con las cuestiones específicas que el investigador pretende estudiar (Branco, 1993).

Además de la necesidad de considerar la dimensión procesual de la producción del conocimiento, la perspectiva sociocultural constructivista apunta otros aspectos fundamentales para el estudio de fenómenos relativos al desarrollo humano. Uno de esos aspectos está relacionado con el papel fundamental que ocupa el lenguaje en el estudio y comprensión de ese desarrollo. El lenguaje representa la realidad psicológica y, al mismo tiempo, participa de su constitución. Así, es importante el estudio de la elaboración discursiva de los sujetos a partir de su contacto con el universo de significados transmitidos y reconstruidos culturalmente. Otro aspecto importante es la necesidad de considerar 
los contextos y prácticas culturales donde los fenómenos estudiados ocurren (Rossetti-Ferreira, Amorin, Soares-Silva \& Oliveira, 2008).

A partir de esos aspectos esenciales para la comprensión de los procesos de desarrollo tomamos la decisión de usar una metodología cualitativa y descriptiva de las concepciones, de un grupo de educadores de la Educación Infantil brasileña, sobre los conflictos interpersonales y sobre su papel en el desarrollo y educación moral de los niños. También fueron investigadas las principales estrategias de intervención que se originan a partir de esas concepciones.

\section{Participantes}

Participaron del estudio nueve educadores de una institución pública de Educación Infantil de Brasilia-DF, Brasil siendo cuatro profesoras del turno matutino, tres profesoras del turno vespertino y dos funcionarios de la dirección de la escuela (director y vicedirectora). Todos los participantes tenían formación de nivel superior y diferentes cursos de extensión o formación continuada, experiencia de trabajo de más de 10 años en la educación, y experiencia de más de cinco años en la escuela. Todos ya habían trabajado con nińos de diferentes edades y consideraban que tenían una experiencia adecuada para lidiar con las cuestiones que surgen en el cotidiano escolar.

\section{Procedimiento}

Los educadores concordaron en participar en una entrevista semiestructurada, realizada de acuerdo con su disponibilidad de tiempo. Las cuatro profesoras del turno matutino concordaron con la realización de observaciones naturalistas en el contexto del aula. La escuela fue seleccionada para el estudio por ser una institución de referencia en Educación Infantil de la Red Pública de Enseñanza de Brasilia-DF, Brasil y fue indicada por la Secretaria de Educación de Brasilia-DF. El equipo pedagógico que participó de la investigación se mostró interesado por el estudio, concordando con la realización del mismo. 
Durante la primera fase de la investigación, fueron realizadas un total de 10 sesiones de observación naturalista como parte del proceso de inmersión etnográfica en el contexto de la escuela y totalizaron 35 horas y 10 minutos de observación. Las observaciones fueron realizadas en los diferentes espacios y horarios de la rutina escolar (horario de entrada, horario del parque, merienda colectiva, actividades en el aula y horario de salida), y tuvieron como objetivo principal realizar una aproximación etnográfica al objeto y contexto de estudio, así como obtener informaciones sobre la dinámica de las interacciones sociales del contexto específico. Cinco de las sesiones fueron realizadas en el aula y fueron grabadas en vídeo después de obtener la autorización de los responsables de los niños. Todas las observaciones fueron registradas en un protocolo de registro, transcripción y análisis elaborado específicamente para la investigación, destacándose las acciones y verbalizaciones de las profesoras y de los niños, así como el tiempo de ocurrencia y la continuidad de las interacciones.

Las sesiones de observación naturalistas registradas en el protocolo de observación fueron analizadas micro genéticamente en función de la importancia y de la adecuación del análisis microgenético para el estudio de las interacciones sociales y del desarrollo humano. El análisis micro genético permite interpretar la comunicación como un proceso dinámico, en el cual todos los participantes desempeñan un papel igualmente importante, resaltando la interdependencia entre los individuos y los diferentes contextos socioculturales (Kelman\& Branco, 2004, 2009). Además de eso, el análisis microgenético posibilita el estudio de la calidad de las interacciones sociales, y también posibilita detectar como esas interacciones facilitan o dificultan los procesos de aprendizaje (Palmieri \& Branco, 2007; Kelman\& Branco, 2004, 2009). En estrecha relación con la calidad de las interacciones sociales, es posible estudiar las negociaciones que ocurren en el flujo interactivo entre profesor-alumno y alumno-alumno.

Para la realización del análisis micro genético, cada sesión transcrita fue dividida en episodios, agrupando un conjunto de interacciones continuas sobre cuestiones relevantes para el estudio dentro de un mismo tema. 
Fue realizado un análisis de contenido de los episodios, resaltando indicadores relacionados a los objetivos de la investigación con la finalidad de facilitar el proceso de inferencia relativo a la posible atribución de sentido a las experiencias e interacciones concretas por parte de los niños en las actividades analizadas (Rey, 1997). Los análisis realizados permitieron definir la calidad de las interacciones profesoras-niños, así como la calidad de los procesos, estrategias y mecanismos educativos usados por las profesoras en los momentos de conflictos interpersonales entre los niños.

Para las entrevistas semiestructuradas fue elaborada una guía a partir de los objetivos del estudio. En la guía fueron abordados tópicos como los conceptos de moral y desarrollo moral, el papel de la escuela en el desarrollo moral, el concepto de conflicto, los tipos de conflictos interpersonales comunes entre los niños, el papel del conflicto en el desarrollo y educación moral, y las principales estrategias educativas usadas a la hora de intervenir en las situaciones de conflicto interpersonal entre los niños. Al final de la entrevista, los participantes analizaron dos situaciones hipotéticas envolviendo conflictos interpersonales. Las situaciones hipotéticas fueron elaboradas a partir de las observaciones del período de inmersión etnográfica, considerando los tipos de conflictos comunes entre los niños del contexto escolar específico. Las entrevistas fueron grabadas en audio y transcritas integralmente.

El análisis del discurso de los educadores fue realizado a partir de categorías analíticas elaboradas de acuerdo con los objetivos de la investigación y con palabras-clave relativas a los temas de la guía. Entre las categorías elaboradas resaltamos las que permitieron analizar las concepciones de los educadores con relación al papel del conflicto interpersonal en el desarrollo y educación moral: papel del conflicto interpersonal en el desarrollo moral; conflictos comunes entre los nińos; principales mecanismos para lidiar con los conflictos; y percepción de la práctica pedagógica en situaciones de conflictos (análisis de las situaciones hipotéticas). 


\section{Resultados}

Según ocho profesionales de la escuela (88\%), los conflictos interpersonales son parte del desarrollo infantil y son importantes para el desarrollo y educación moral de los niños, como ilustran los comentarios de tres entrevistas realizadas con las profesoras:

Porque..., asi..., esos conflictos, ellos generan crecimiento. Lógico que no es eso..., esa cosa... que pasa todos los días en la media, esa cosa así.. Pero, aquel..., que... que tuvo una crisis por causa de un juguete. No quiere prestarlo y otro que no quiere ceder. ¿Entiende? Eso trae crecimiento, madurez para ellos. (Profesora del grupo de $2^{\circ}$. Período, turno matutino).

Yo creo que es importante porque eso estructura. Asi, ese estar social del niño. 'Me tocaba a mi..., 'Fulano no respetó,' 'No quiso jugar más', ¿Y ahora? ¿Cómo va a ser? ¿Cómo yo me voy a imponer delante de eso?' ¿Qué alternativa voy a dar?' (la profesora habla como un niño hablando/pensando). A mi eso me parece muy estructurarte, hasta por el papel social de cada uno. (Profesora del grupo de $2^{\circ}$. Periodo, turno vespertino).

Bueno, a mi me parece natural y normal. Porque es una fase en que ellos están descubriendo, que ellos están aprendiendo. Y esos conflictos, yo creo que tienen un valor muy grande en la vida de ellos porque ellos aprenden a defenderse, ellos aprenden a resolver sus propios conflictos. Y... hay muchos adultos que no consiguen hacer eso, porque muchos, algunas veces, ni convivian con otros niños, estaba muy restricto al mundo familiar de él, y no tenía, en esa edad, con quien tener conflictos. Yo creo que esos atritos..., ellos aprenden mucho. (Profesora del grupo de 3er. Periodo del turno vespertino).

Según los educadores, en el contexto de los conflictos interpersonales los niños aprenden a interaccionar con el otro, así como a compartir y resolver las diferencias personales. Los conflictos más comunes son aquellos que surgen en función de los objetos (juguetes y materiales pedagógicos), del espacio y de diferencias relativas a objetivos y deseos personales (realizar juegos y actividades diferentes). Aunque sea importante 
para el desarrollo, el conflicto pasa a tener una connotación negativa porque puede ser resuelto a través del confrontamiento físico (mordidas, empujones), lo que es común entre los niños pequeños. En función de eso, los conflictos aparecieron en la elaboración discursiva de los profesionales como situaciones generadoras de angustia, que deben ser evitadas de varias formas sea controlando la conducta de los niños a través de las sanciones y de las reglas establecidas, o interfiriendo antes que los conflictos se establezcan, como ilustran las respuestas de dos profesoras:

Es lo siguiente: de acuerdo con lo que nosotros acordamos al iniciar el año, de no pegarle al otro, no pelear, yo los coloco separados del resto del grupo y los pongo a conversar entre ellos para llegar a un acuerdo. Porque si ellos no llegan a un acuerdo, ahi el castigo es para los dos. Si ellos no siguen la regla, ahi ellos se van aquedar de castigo. ¿Cuál es el castigo de ellos? No ir al parque un día o no ir para la casita a jugar, no ir a la actividad que tenemos en el patio, que a ellos les encanta..., les encantan esos juegos. Entonces..., ellos intentan conversar y llegar a un acuerdo... (Profesora del grupo de 3er. Periodo del turno matutino).

Lo que yo hago son mediaciones... en los situaciones de conflictos entre ellos, situaciones así... Ahi los separo... cuando yo ya sé que va a suceder algo, separo... Tengo que ser muy rápida, hasta porque ellos se pueden hacer daño. Ahi, generalmente, yo los cambio de lugar, separo. Si está en el medio de la fila y peleó, ahi yo pongo uno para frente y otro para atrás, separo... Yo creo que es mejor separar. Hay conflictos que se pueden resolver..., asi, de una forma más dura... retirarlos de la actividad o llamar a la familia. Si converso una vez y el niño continua insistiendo, entonces tenemos que conversar con la familia, porque yo creo que la familia tiene que participar junto con nosotros. (Profesora del grupo de $1^{\circ}$. Período del turno vespertino).

Para cuatro de los profesionales, el conflicto es un reflejo de la falta de límites de la familia, así como de ejemplos inadecuados en el contexto familiar. Para tres de los otros educadores es un reflejo de la agresividad de algunos nińos, que por cualquier cosa entran en conflicto físico con el otro. Ejemplos de esas concepciones sobre el conflicto son las respuestas de dos profesoras y de la vicedirectora de la escuela: 
Mira, yo veo que hay algunos niños que son agresivos, ¿si?? Hay muchas peleas asi, porque hay esos niños que son agresivos. Que por nada pelean $y$ le pegan a los otros. No, no hay conflictos por nada... Asi, por nada, por nada. Cuando uno mira... pueden estar sentados uno al lado del otro, se viran y le pegan al otro. A veces, es el mismo niño... Y el otro va a reaccionar pegando también porque nadie está aqui para aguantar ese tipo de cosas, ¿¿í? Entonces ese es el caso más común (Profesora del grupo de $2^{\circ}$. Período del turno matutino).

¡Bueno! ¡Conversar, conversar! ¡Ellos hablan muy alto! Y conversan mucho, hablan muy alto, a veces, algunos alumnos dicen malas palabras. Entonces asi, conflictos en mi clase... no hay muchos. Ellos tienen lo que traen de la casa. Yo creo que no hay mucho..., asi, disciplina, no tienen muchos limites, los padres no saben imponer limites, no saben educar. (Profesora del grupo de 3er. Período del turno vespertino).

Yo creo que... los niños, ellos tienen..., así, reflejan mucho lo que ellos están pasando dentro de la casa. Entonces, hay una hora en que uno... yo les digo a las profesoras que si uno tiene un poco de conocimiento sobre lo que pasa en la casa, uno tiene una visión, una comprensión mayor de las dificultades que ellos están pasando y muchas veces uno va a ser más sensible y ver que no es algo que sucede gratuitamente, que él está reproduciendo cosas que él está pasando. (Vicedirectora).

De forma general, los profesionales iniciaron su discurso hablando de la importancia del conflicto para el desarrollo y educación moral del niño, pero terminaron afirmando una visión negativa del mismo. Esa visión negativa también apareció en las interacciones concretas de las profesoras con los niños. En las sesiones de observación fueron registradas intervenciones cargadas de emotividad, por parte de las profesoras. Frases como “iiNo!! iiNo!!”, “Que cosa más fea! jMuy fea!”y “i Mira para mi! ;Yo dije que no es para hacer eso!" acabaron comunicándole a los nińos la angustia del adulto delante de la situación de confrontamiento entre ellos, así como su desaprobación y decepción.

De acuerdo con varios autores (Killen \& Nucci, 1995; De Vries \& Zan, 1998; Valsiner \& Cairns, 1992), las situaciones de conflictos interpersonales pueden ser oportunidades fundamentales para el desarrollo. Cuando los conflictos interpersonales son resueltos a través 
del confrontamiento y de la agresión física, los mismos demandan una atención especial por parte de los educadores en función de los riesgos en términos de seguridad para los niños. Entretanto, 'atención especial' no significa eliminar la posibilidad de los conflictos, controlando las acciones e interacciones de los niños (Galvão, 2004). Esos conflictos deben ser trabajados, con la mediación de los adultos, sea incentivando a los niños a conversar para encontrar formas de resolver el conflicto (“PPor qué no conversas con él?”, en Branco, 1989), sea dando ejemplos de resolución constructiva de las divergencias.

Sobre la cuestión anterior, De Vries y Zan (1998) señalan la importancia de la actitud del profesor frente a los conflictos interpersonales de los niños. Las autoras resaltan que el profesor debe mantener una actitud calma frente al estado de perturbación y de descontrol emocional que los niños pueden exhibir en algunos momentos. La tranquilidad del adulto a la hora de resolver los conflictos interpersonales que envuelven confrontamiento físico puede ser percibida, por los niños, como un apoyo en la conducción de sus dificultades. Eso no significa, obviamente, que el profesor no deba evitar activamente dańos físicos entre los nińos.

La visión negativa del conflicto también estuvo presente en las descripciones de las principales estrategias de intervención en los momentos de conflicto. De acuerdo con siete de los entrevistados (77\%), cuando los conflictos son resueltos a través del confrontamiento físico es necesario retirar a los niños de la actividad para que piensen sobre sus acciones. La sanción fue la estrategia de intervención más resaltada en las entrevistas, siendo que también la observamos en algunos episodios analizados a través de hablas e intervenciones como “iTú no le puedes pegar al colega y tienes que dividir! ¡Si no haces eso, entonces no puedes participar en el juego!". De acuerdo con los educadores, la sanción más eficiente para eliminar el conflicto es la retirada del niño de la actividad, sobretodo si esa actividad genera placer. Con el objetivo de ilustrar mejor esa cuestión, colocamos la respuesta de la profesora del grupo de 3er. Período del turno matutino delante de una situación hipotética presentada en la entrevista, en la cual un grupo de nińos había rayado los dibujos de un mural, a la hora de salir para el recreo: 
...Entonces, yo voy a conversar con todos y ellos van a usar el tiempo del recreo para ellos... Como yo te dije, yo soy bien enérgica. Yo regreso con ellos y el tiempo del recreo... yo lo voy a usar para, justamente, llevarlos a percibir que lo que hicieron está errado. ¡Ellos van a perder el recreo!

Además de la sanción, los profesionales de la escuela resaltaron otras estrategias, que consideran importantes. Dejar que los niños resuelvan sus diferencias, conversar con los niños sobre lo ocurrido, resaltar las consecuencias negativas del confronto físico y solicitar una forma de compensación fueron las estrategias de intervención descritas por el grupo de entrevistados. Entretanto, esas estrategias no fueron concretamente observadas en los episodios de conflictos registrados y analizados en el estudio. También no estuvieron presentes en los análisis realizados por los entrevistados en relación a las dos situaciones hipotéticas presentadas en las entrevistas.

En las sesiones de observación, tuvimos la oportunidad de analizar las intervenciones de las profesoras en diferentes tipos de conflicto. Esas intervenciones estuvieron caracterizadas por la asimetría en la interacción profesora-niño, dejando poco o ningún espacio de participación activa por parte de los nińos en la resolución de sus propios conflictos interpersonales. En los episodios de conflictos analizados, las profesoras asumieron una posición central en su aparente resolución, recordando reglas previamente definidas y estableciendo la posibilidad de una sanción. De forma general, los niños no tuvieron la posibilidad de expresarse verbalmente y de forma activa en relación a sus propios conflictos, sugiriendo o negociando estrategias de resolución a partir de sus propias necesidades y percepciones de la situación.

La participación activa de los niños en sus propios conflictos es fundamental para el desarrollo moral (Branco, 2003; Branco \& Mettel, 1995; De Vries \& Zan, 1998). Cuando los niños tienen esa oportunidad, ellos pueden colocar sus puntos de vista y percepciones sobre lo ocurrido, así como negociar y construir en conjunto estrategias de solución adecuadas para todas las partes del conflicto. Entretanto, es importante resaltar que no son todos los niños los que consiguen resolver siempre sus conflictos interpersonales solos, siendo necesario e 
importante la mediación del adulto. Es decir, el adulto en la posición de un 'compañero' con más experiencia puede ayudar a los niños en la resolución de los conflictos, a partir de las posibilidades reales que los mismos tienen, promoviendo, así, el aprendizaje y el desarrollo de nuevas estrategias de solución.

En dos de los episodios analizados observamos algunos niños solicitando la mediación de las profesoras para resolver conflictos interpersonales. En respuesta a la demanda de los niños, las profesoras intervinieron recordando reglas establecidas con anterioridad o dando una solución acabada, sin la participación de los niños. Intervenciones que acabaron reforzando la dependencia de los niños a la hora de resolver conflictos con sus colegas. El hecho de que los niños hayan solicitado la intervención de las profesoras puede ser considerado como un indicador de pocas habilidades o estrategias para resolver la situación sin el auxilio del adulto. Siendo así, es importante la mediación del adulto en el sentido de ayudar al niño a buscar estrategias de resolución y desarrollar habilidades interpersonales diferentes de transferir la responsabilidad para una figura de autoridad. Datos relativos a investigaciones sobre las interacciones niño-niño en la Educación Infantil apuntan para la eficiencia de intervenciones de la profesora tales como “¡Vamos a conversar con él!”, o entonces “Bueno, ¿y cómo ustedes pueden resolver eso? ¿Qué es lo que ustedes pueden hacer ahora? (Branco, 1989; Tobin, Wu \& Davidson, 1989).

De forma general, podemos afirmar que a pesar de que los profesionales de la escuela hayan colocado el conflicto interpersonal como un elemento importante del desarrollo y educación moral de los nińos, partes de su propia elaboración discursiva y las acciones concretas de las profesoras observadas en los momentos de conflicto apuntan una visión negativa del mismo y una tendencia a inhibirlo. Las intervenciones concretas en los momentos de conflicto se orientaron para su eliminación y no para su resolución, en detrimento de la posibilidad de aprovecharlo como una oportunidad de construcción conjunta y desarrollo de habilidades de negociación, capacidades de diálogo, interacción y convivencia respetuosa entre los niños. 


\section{Consideraciones finales}

La educación actual está permeada de ideas innovadoras que todavía no fueron incorporadas de forma plena en el cotidiano de las instituciones educativas. La escuela, a pesar de tener un discurso innovador, tiene una práctica pautada por los principios que orientan la educación tradicional, colocando en segundo plano el proceso de socialización y el desarrollo y educación de aspectos importantes como la moralidad. Eses resultados no son exclusivos de nuestra investigación, han surgido en otros estudios (e.g. Manzini, 2013; Lima 2000; Padilha, 2010; Palmieri, 2003; Salomão, 2001) que apuntan así la necesidad de discusiones y estrategias que objetiven la transformación concreta de las prácticas educativas y la reflexión sobre la formación inicial y continuada de los profesores y sobre los objetivos reales de la educación.

En el caso específico del papel de los conflictos interpersonales para el desarrollo y educación moral, se muestra como necesario un cambio en la percepción real que los educadores tienen. Como vimos en la investigación realizada, hay un predominio de una visión negativa del conflicto interpersonal. Aunque esa visión negativa aparezca con menor intensidad en la elaboración discursiva de los educadores investigados, ella apareció de forma más clara en las intervenciones pedagógicas que acostumbran ocurrir en el cotidiano escolar. Intervenciones pedagógicas cargadas de emotividad y que tienen por objetivo eliminar el conflicto, estableciendo sanciones y/o disminuyendo la posibilidad de que los niños ejerzan sus posibilidades de construcción de la autonomía y asertividad.

En el contexto educativo brasileño, autoras como Galvão (2004) y Vinha $(2004,2009)$ también resaltan la visión negativa del conflicto, por parte de los profesores. Galvão (2004) estudió los tipos de conflictos y las estrategias de intervención en el contexto de una escuela de Educación Infantil brasileña. De acuerdo con la autora, la visión negativa del conflicto, por parte de las profesoras de la escuela analizada, está presente en las diferentes estrategias de resolución e intervención que surgen en el cotidiano escolar. Esas estrategias de resolución e intervención generalmente están cargadas de tensión lo que dificulta la distinción 
de los conflictos constructivos, que indican y promueven el crecimiento, de los conflictos no constructivos, que indican estagnación y se confunden con la violencia. Vinha (2004) estudió el proceso de resolución de los conflictos interpersonales en el contexto de escuelas autocráticas (con una concepción tradicional y negativa de los conflictos) y en escuelas democráticas (con una concepción constructivista de los conflictos), estableciendo una posible relación entre el ambiente de las escuelas y el desarrollo de los niños en relación a los niveles de entendimiento personal conforme Selman (1980). Según la autora, en el ambiente escolar autocrático fue encontrado menor desarrollo del entendimiento personal entre los niños. Ya en el ambiente escolar democrático, fue constatado, en las relaciones entre los niños, un mayor desarrollo del entendimiento personal. Para Vinha (2004), los datos de la investigación muestran como las concepciones del conflicto que predominan en la escuela y el ambiente socio-moral de la misma pueden tener un impacto en el proceso de desarrollo y socialización de los educandos.

En el contexto educativo español, Jares (2006) estudió la percepción que alumnos y profesores de centros de enseñanza de Galicia (Espańa) tenían sobre los conflictos. De acuerdo con el autor, tanto los alumnos como los docentes presentaron una percepción altamente negativa del conflicto. Esa percepción negativa tenía como base la estrecha relación que los participantes establecían entre los conceptos de conflicto, violencia e indisciplina. Para ellos, las situaciones de conflicto pueden generar espacio para la indisciplina y relaciones de violencia entre alumno-alumno y alumno-profesor y así deben ser evitadas a cualquier costo. A partir de los resultados, Jares (2006) resalta que esa visión negativa del conflicto acaba por disminuir considerablemente la posibilidad de verlo como un hecho educativo y una oportunidad de aprendizaje de padrones de interacción social que permitan una buena convivencia social, siendo que esta última es apuntada como necesaria y deseable tanto por los alumnos como por los docentes.

También es importante decir que los participantes de la investigación de Jares (2006) resaltaron, como una de las principales causas de los conflictos y de la violencia, el ambiente desestructurado de la familia, 
lo cual coincide con los datos del presente trabajo. Asimismo, cuatro de los profesionales entrevistados señalaron una posible relación entre el ambiente familiar y los conflictos interpersonales de los niños en el contexto escolar. El ambiente familiar puede influenciar en la ocurrencia de conflictos porque muchas familias son permisivas y no colocan límites claros para los niños. Además de eso, problemas como desentendimiento entre los padres, falta de cariño en el contexto familiar y ejemplos de acciones inadecuadas por parte de los padres pueden llevar al niño a desentenderse con otras personas en el contexto escolar.

Jares (2006) explica que existe una contradicción entre la necesidad del conflicto para formar patrones de interacción social con base en el respeto y la tendencia de los profesores a eliminar los conflictos por causa de fricciones físicas y verbales. Concordamos con las colocaciones del autor y resaltamos la necesidad de retomar la obra de varios autores constructivistas (e.g. DeVries y Zan, 1998; Kolhberg, 1981; Piaget, 1932/1994; Selman, 1980) que señalan el papel fundamental del conflicto para el desarrollo de la moralidad, una vez que los mismos permiten la construcción de estrategias de entendimiento interpersonal. De acuerdo con Piaget (1932/1994), los conflictos interpersonales entre los niños desencadenan un desequilibrio interno que los lleva y motiva a procurar nuevas maneras y posibilidades de establecer la relación con el otro. Para Piaget (1932/1994), los conflictos interpersonales facilitan que el niño empiece a considerar otros puntos de vista, sentimientos y diversas formas de resolución.

Según De Vries y Zan (1998), el conflicto interpersonal es un espacio primordial para que el nińo tome consciencia de que los otros tienen opiniones, necesidades y deseos diferentes de los suyos, así como de las posibilidades de coordinar sus propias perspectivas con las perspectivas de los otros, construyendo un espacio de entendimiento interpersonal que permita mantener la reciprocidad en las relaciones e interacciones sociales. El conflicto interpersonal es una situación propicia para pensar sobre cómo se puede proceder en situaciones y cuestiones que dan margen a diferentes puntos de vista, necesidades, objetivos y sentimientos. 
Además de la investigación comentada anteriormente, Jares (2002) coordinó y realizó un estudio en 118 centros de Enseñanza Fundamental de los sectores público y privado de toda Galicia, España, sobre la formación de los profesores en relación a los temas que nos ocupan. Los datos empíricos del estudio evidenciaron que los profesionales de la educación, que tratan cotidianamente con todo un conjunto de aspectos y de situaciones fundamentales para la construcción y/o formación de habilidades sociales que objetivan la convivencia armónica y democrática entre las personas, no tienen formación para lidiar con situaciones de conflictos. En la investigación realizada por Jares (2002), 67,6\% de los 1131 docentes participantes alegaron no haber recibido ningún tipo de formación inicial sobre cómo lidiar con cuestiones que envuelven la buena convivencia y, específicamente, con la resolución de situaciones de conflicto que surgen en el contexto de las interacciones y relaciones interpersonales, siendo este un tema fundamental para el desarrollo de la moralidad. Según el autor, esa falta de formación contradice los objetivos actuales de la educación que debe apoyar la convivencia democrática y pacífica en el contexto educativo formal.

La contradicción tiene como base el modelo positivista y la educación formal tradicional que han reducido el papel de la escuela y de los profesores al proceso de escolarización. O sea, el modelo positivista, que sustenta la mayoría de los sistemas educativos, lleva a la escuela a centrarse en el cumplimiento de obligaciones y objetivos que enfatizan los contenidos académicos. En nuestra investigación, todos los educadores entrevistados señalaron la falta de formación inicial y continuada sobre el tópico. Siendo así, aprovechamos para resaltar la importancia de la formación continuada de los profesores para la promoción del desarrollo moral en el contexto educativo. La psicología escolar tiene un papel fundamental en esa formación continuada, como enfatizan Barrios, Marinho-Araújo y Branco (2011).

Como enfatizamos a lo largo del texto, los profesores y la escuela tienden a eliminar los conflictos interpersonales, aunque estos sean un espacio privilegiado y natural para la construcción de padrones de interacción social adecuada para una convivencia armónica y democrática. 
La eliminación de los conflictos interpersonales también elimina un espacio natural de formación de los valores sociomorales. En ese contexto escolar, caben las siguientes preguntas: ¿Cuál es el espacio que los niños tienen para construir esos valores e habilidades? ¿Cuál es el espacio para aprender a negociar os conflictos y diferencias?

La visión negativa con relación al conflicto interpersonal no quita la importancia del mismo para la educación y para el desarrollo de la moralidad. Siendo así, es fundamental realizar nuevas investigaciones sobre el tema, sobretodo en relación a las estrategias participativas que pueden ser implementadas en el contexto escolar. También son necesarias nuevas investigaciones sobre las posibles estrategias y programas de formación inicial y continuada de los educadores. Esos programas deben contemplar no solo el desarrollo moral de los estudiantes, sino también su desarrollo integral. Recordamos que los objetivos de la educación en la actualidad buscan la formación de personas y ciudadanos comprometidos éticamente con la construcción de una sociedad más democrática.

\section{Referencias}

Barrios, A. (2009). Desenvolvimento moral e práticas pedagógicas na educação infantil: Um estudo sociocultural construtivista. Dissertação de Mestrado, Instituto de Psicologia, Universidade de Brasília, Brasil.

Barrios, A. (2013). Diálogo ou heteronomia no ensino fundamental? Desenvolvimento moral, cultura e práticas educativas. Tese de Doutorado, Instituto de Psicologia, Universidade de Brasília, Brasil.

Barrios, A., Marinho-Araujo, C. M. \& Branco, A. U. (2011). Formação continuada do professor: desenvolvendo competências para a promoção do desenvolvimento moral. Revista Psicologia Escolar e Educacional, 15(1), 91-99. http://dx.doi.org/10.1590/S141385572011000100010 
Bellini-Leite, S. C., Vargas, P. R. \& Ireno, E. M. (2012). A importância das relações interpessoais satisfatórias: Uma revisão da literatura sobre habilidades sociais. CES Revista - Juiz de Fora, 26(1), 273288.

Branco, A. U. (1989). Socialização na pré-escola: O papel da professora $e$ da organização das atividades no desenvolvimento de interaçóes sociais entre crianças. Tese de Doutorado, Instituto de Psicologia da Universidade de São Paulo, Brasil.

Branco, A. U. (1993). Sociogênese e canalização cultural: Contribuiçóes à análise do contexto das salas de aula. Temas em Psicologia, 3, 9-17.

Branco, A. U. (2003). Social development in cultural context: Cooperative and competitive interaction patterns in peer relations. En J. Valsiner y K. J. Connolly (Orgs.), Handbook of developmental psychology (Vol. 1, pp. 238-256). London: Sage.

Branco, A. U. (2009). Cultural practices, social values, and childhood education. En M. Fleer, M. Hedegaard, J. Tudge, A. Prout (Orgs.), World Year Book of Education 2009: Childhood studies and the impact of globalization: Policies and Practices at Global and Local Levels (pp. 44-66). New York: Routledge.

Branco, A. U. (2012). Values and sociocultural practices: Pathways to moral development. En J. Valsiner (Ed.), The Oxford Handbook of culture and psychology (pp. 1833-1880). New York: Oxford University Press. http://dx.doi.org/10.1093/ oxfordhb/9780195396430.013.0036

Branco, A. U. \& Barrios, A. (2014). Desarrollo moral y ontogénesis de los valores democráticos en la escuela. En F. G. Londra y A. R. Rivero (Orgs.), Hacer(se) ciudadan@s: Una Psicología para la democracia. (pp. 187-213). Buenos Aires: Miño y Dávila.

Branco, A. U. \& Mettel, T. (1995). O processo de canalização cultural das interaçóes criança-criança na escola. Psicologia: teoria e pesquisa, 11(1), 13-22.

Branco, A. U. \& Valsiner, J. (1997). Changing methodologies: A co-constructivist study of goal orientations in social interactions. 
Psychology and Developing Societies, 9(1), 35-64. http://dx.doi. org/10.1177/097133369700900103

Buhs, S., Ladd, G. W. \& Herald, S. H. (2006). Peer exclusion and victimization: Processes that mediate the relation between peer group rejection and children's classroom engagement and achievement? Journal of Educational Psychology, 98, 1-13. http:// dx.doi.org/10.1037/0022-0663.98.1.1

Corsi, B. R. (2011). Relações e conflitos entre crianças na Educação Infantil: $\mathrm{O}$ que elas pensam e falam sobre isso. Educar em Revista, (42), 279-296.

DeVries, R. \& Zan, B. (1998). A ética na educação infantil: o ambiente sócio-moral na escola. Porto Alegre: Artes Médicas.

DeVries, R. \& Zan, B. (2003). When children make rules. Educational Leadership: Building Classroom Relationship, 61(1), 64-67.

Fleer, M., Hedegaard, M. \& Tudge, J. (2009). Childhood studies and impact of globalization: Policies and practices at global and local levels. Londres: Routledge.

Freitag, B. (1997). Itinerários de Antígona: a questão da moralidade. Campinas: Papirus.

Frick, L. T. (2011). As relaçóes entre os conflitos interpessoais e o bullying: Um estudo nos anos iniciais do Ensino Fundamental de duas escolas públicas. Dissertação de Mestrado em Educação. Faculdade de Ciências e Tecnologia, Universidade Estadual Paulista "Júlio de Mesquita Filho", Presidente Prudente, São Paulo.

Galvão, I. (1995). Henri Wallon: Uma concepção dialética do desenvolvimento infantil. Petrópolis, Rio de Janeiro: Vozes.

Galvão, I. (2004). Cenas do Cotidiano Escolar: Conflitos sim, violência náo. Rio de Janeiro: Vozes.

Hoffman, M. L. (2000). Empathy and moral development: Implications for caring and justice. New York: Cambridge University Press. http://dx.doi.org/10.1017/CBO9780511805851

Jares, X. R. (2002). Aprender a convivir. Revista Interuniversitaria de Formación del Profesorado, 44, 79-92. 
Jares, X. R. (2006). Conflicto y convivencia en los centros educativos de secundaria. Revista de Educación, 339, 467-491.

Kelman, C. A. \& Branco, A. U. (2004). Análise microgenética em pesquisa com alunos surdos. Revista Brasileira de Educação Especial, 10(1), 93-106.

Kelman, C. \& Branco, A. U. (2009). (Meta)Communication strategies in inclusive classes for deaf students. American Annals of the Deaf (Washington, D.C.), 154, 371-381. http://dx.doi.org/10.1353/ aad.0.0112

Killen, M. \& Nucci, L. P. (1995). Morality, autonomy, and social conflict. En M. Killen y D. Hart (Orgs.), Morality in everyday life: Development perspectives (pp. 52-86). Cambridge: Cambridge University Press.

Kohlberg, L. (1981). The Psychology of Moral Development. New York: Harper Row.

Kolominskii, I. L. \& Zhiznevskii, B. P. (1992). A sociopsychological analysis of conflicts among children during play. Journal of Russian and East European Psychology, 30(5), 72-86. http:// dx.doi.org/10.2753/RPO1061-0405300572

Ladd, G. W. \& Troop-Gordon, W. (2003). The role of chronic peer difficulties in the development of children's psychological adjustment problems. Child Development, 74(5), 1344-1367. http://dx.doi.org/10.1111/1467-8624.00611

Lewin, L. (1935). A dynamic theory of personality: Select papers. New York: McGraw-Hill.

Licciardi, L. M. S. (2010). Investigando os conflitos entre as crianças na escola. Dissertação de Mestrado, Faculdade de Educação, Universidade Estadual de Campinas, Brasil.

Lima, M. S. M. (2000). Silêncio elou participação? A questão da disciplina na concepção e na prática de professoras da $1^{a}$. Série do Ensino Fundamental. Dissertação de Mestrado, Instituto de Psicologia da Universidade de Brasília, Brasília.

Manzini, R. P. (2013). Bullying no context escolar: Prevenção da violência e promoçáo da cultura de paz na perspectiva de adultos e crianças. 
Tese de Doutorado, Instituto de Psicologia, Universidade de Brasília, Brasil.

Martins, L.C. \& Branco, A.U. (2001). Desenvolvimento moral: Consideraçóes teóricas a partir de uma abordagem sociocultural construtivista. Psicologia: Teoria e Pesquisa, 17(2), 169-176. http://dx.doi.org/10.1590/s0102-37722001000200009

Martins, G.C. \& Garcia, A. (2011). Conflito interpessoal entre brasileiros e entre brasileiros e estrangeiros em empresas multinacionais de Manaus. Cadernos de Psicologia Social do Trabalho, 14(2), 179-194. http://dx.doi.org/10.11606/issn.1981-0490. v14i2p179-194

McIntyre, S.E. (2007). Como as pessoas gerem o conflito nas organizaçôes: Estratégias individuais. Análise Psicológica, 2(XXV), 295-305.

Oetzel, J., Dhar, S. \& Kirschbaum, K. (2007). Intercultural conflict from a multilevel perspective: trends, possibilities, and future directions. JournalofIntercultural Communication Research, 36(3), 183-204. http://dx.doi.org/10.1080/17475750701737124

Padilha, M. (2010). Negritude e infância. 212 f. Dissertação de Mestrado, Instituto de Psicologia, Universidade de Brasília, Brasil.

Palmieri, M.W.A. (2003). Cooperação, competiçāo e individualismo: Uma análise microgenética de contextos de desenvolvimento na pré-escola. Tese de Doutorado, Instituto de Psicologia da Universidade de Brasília, Brasília.

Palmieri, M. W.A. \& Branco, A. U. (2007). Educaçáo infantil, cooperação e competiçâa: Análise microgenética sob uma perspectiva sociocultural. Psicologia Escolar e Educacional, 11, 365-378. http://dx.doi.org/10.1590/S1413-85572007000200014

Paolichi, P. (2007). The institutions inside: self, morality, and culture. En J. Valsiner y A. Rosa (Eds.), The Cambridge Handbook of sociocultural psychology (pp. 560-575). New York: Cambridge University Press. http://dx.doi.org/10.1017/ CBO9780511611162.030 
Piaget, J. (1994). O juizo moral na criança. São Paulo: Summus. (Originalmente publicado em 1932).

Piaget, J. (2006). Seis estudos de psicologia. Rio de Janeiro: Forense Universitária. (Originalmente publicado em 1964).

Ratner, C. (2002). Cultural psychology: Theory and method. New York: Plenum. http://dx.doi.org/10.1007/978-1-4615-0677-5

Rey, F. G. (1997). Epistemología cualitativa y subjetividad. São Paulo: Educ.

Rey, F. G. \& Martínez, A. M. (1989). La personalidad, su educación y desarrollo. La Habana: Pueblo y Educación.

Rogoff, B. (2005). A natureza cultural do desenvolvimento humano. Porto Alegre: Artmed.

Rossetti-Ferreira, M.C., Amorin, K. S., Soares-Silva, A. P. \& Oliveira, Z.M.R. (2008). Desafios metodológicos na perspectiva da rede de significações. Cadernos de Pesquisa, 38(133), 147-170. http:// dx.doi.org/10.1590/S0100-15742008000100007

Salomão, S. J. (2001). Motivação social: Comunicação e metacomunicação na co-construção de crenças e valores no contexto de interaçóes professora-alunos. Dissertação de Mestrado, Instituto de Psicologia da Universidade de Brasília, Brasília.

Sanchez-Burks, J., Nisbett, R. E. \& Ybarra, O. (2000). Cultural styles, relational schemas and prejudice against outgroups. Journal of Personality and Social Psychology, 79(2), 174-189. http://dx.doi. org/10.1037/0022-3514.79.2.174

Selman, R. (1980). The growth of interpersonal understanding. New York: Academic Press.

Ting-Toomey, S. (1988). Intercultural conflicts: a face-negotiation theory. En Y. Kim y W. Gudykunst (Eds.), Theories in intercultural communication (pp. 213-235). Newbury Park, CA: Sage.

Tobin, J. J., Wu, D. Y.H. \& Davidson, D. H. (1989). Preschool in three culture: Japan, China and the United States. New Haven: Yale University Press.

Valsiner, J. (1989). Human development and culture: The social nature of personality and its study. Lexington, MA: Lexington. 
Valsiner, J. (2012). Fundamentos da Psicologia Cultural: Mundos da mente, mundos da vida. Porto Alegre: Artmed.

Valsiner, J. \& Cairns, R. (1992). Theoretical perspectives on conflict and development. En C.V. Shantz y W. W. Hartup (Eds.), Conflict in child and adolescent development (pp. 15-35). Cambridge, UK: Cambridge University Press.

Vinha, T. (2004). O processo de resolução dos conflitos interpessoais na escola autocrática e democrática. Revista da Faculdade Adventista da Bahia "Formadores: Vivencias e Estudos" (Cachoeira), 1(1), 63-80.

Vinha, T. (2009). Autoridade Autoritária. Revista Nova Escola on-line, Disponível em: http://revistaescola.abril.com.br/formacao/ autoridade-autoritaria-504466.shtml?page=1 Acceso: 28 de marzo de 2016.

Vygotsky, L.S. (1984). A formação social da mente. São Paulo: Martins Fontes.

Vygotsky, L. S. (2004). Psicologia pedagógica. Sáo Paulo: Martins Fontes. Wallon, H. (2007). A evolução psicológica da criança. São Paulo: Martins Fontes. (Originalmente publicado en 1941).

Recibido el 29 de diciembre, 2014

Aceptado el 11 de abril, 2016 